\title{
Revisión sistemática del efecto de las funciones ejecutivas en el rendimiento académico
}

\author{
María Mónica Montes Miranda \\ al365408@uji.es \\ Raquel Flores Buils \\ flores@uji.es \\ Clara Andrés Roqueta \\ candres@uji.es
}

\section{Resumen}

La neurociencia educativa es un campo de investigación interdisciplinar que busca traducir los resultados de la investigación en neurociencia, para comprender los efectos de la educación en el cerebro y mejorar las prácticas de enseñanza-aprendizaje en la escuela. En este campo, en las últimas dos décadas, se ha generado un creciente interés científico en el estudio del desarrollo de las funciones ejecutivas (FE) durante el periodo escolar. La evidencia de que la intervención y el entrenamiento de estos procesos puede generar repercusiones positivas en el rendimiento académico (RA) ha estimulado una variada producción de información en los últimos diez años. Esta revisión pretende aunar aquellas investigaciones en las que se vinculan las variables de RA y FE, realizadas entre los años 2000 y 2018, en las bases de datos Proquest, Pubmed y Dialnet, con el propósito de conocer el estado actual de este campo del conocimiento. Para esta revisión se han seguido las directrices de la declaración PRISMA. Los resultados muestran cuáles son los autores más prolíficos en este campo, así como los tipos de investigación más utilizados, los años y países más productivos y los principales hallazgos en este campo. A la luz de estos resultados, se concluye que hay una escasez de estudios teóricos, lo que hace necesario establecer un modelo teórico unificador e incrementar los estudios experimentales planteados desde distintos diseños de investigación, que aporten diferentes perspectivas al conocimiento de este ámbito.

Palabras clave: funciones ejecutivas, rendimiento académico, neurociencia educativa, revisión sistemática. 


\begin{abstract}
Educational neuroscience is an interdisciplinary field of research that seeks to translate the results of neuroscience research, to understand the effects of education on the brain and to improve teaching-learning practices at school. In this field, in the last two decades there has been a growing scientific interest in the study of the development of executive functions (EF) during the school period. The evidence that the intervention and training of these processes can generate positive repercussions on academic performance (AP), has stimulated a varied production of information in the last ten years. This review aims to combine those researches in which the AP and EF variables, made between 2000 and 2018, are linked in the Proquest, Pubmed and Dialnet databases, with the purpose of knowing the current state of this field of knowledge. The guidelines of the PRISMA Declaration have been followed for this review. The results show which are the most prolific authors in this field, as well as the most used types of research, the most productive years and countries and the main findings in this field. In the light of these results, it is concluded that there is a shortage of theoretical studies, which makes it necessary to establish a unifying theoretical model, and to increase the experimental studies raised from different research designs, which contribute different perspectives to the knowledge of this field.
\end{abstract}

Keywords: executive functions, academic performance, educational neuroscience, systematic review.

\title{
Introducción
}

En las últimas dos décadas se ha generado un creciente interés científico en el estudio del desarrollo de las funciones ejecutivas durante el periodo escolar. La evidencia de que la intervención y el entrenamiento de estos procesos puede generar repercusiones positivas en el rendimiento académico ha dejado ver la necesidad de seguir investigando en este campo para explicar si la estimulación directa de los componentes de las funciones ejecutivas conlleva a una mejora efectiva del rendimiento académico.

\section{Funciones ejecutivas y su concepto}

El término funciones ejecutivas fue introducido por Lezak (1983), aunque fue Luria (1986) quien resaltó por primera vez la importancia del área prefrontal como estructura capaz de controlar las conductas y los procesos de pensamiento más complejos.

Según Lezak (1983), las FE se refieren a la capacidad del ser humano para formular metas, planificar objetivos y ejecutar conductas de un modo eficaz. Por tanto, la finalidad última de las mismas es mantener el control de la conducta.

Así pues, el sistema ejecutivo guarda una relación más estrecha con actividades intencionales, novedosas y no rutinarias, que exigen inhibir las respuestas habituales, requieren planificación y toma de decisiones y precisan de flexibilidad mental y atención sostenida (Lezak 1983; Stuss 1992). Las funciones ejecutivas son necesarias para programar las acciones dirigidas al logro de un objetivo de un modo eficiente y 
para resolver problemas complejos, ya que gracias a su carácter supramodal son capaces de supervisar el resto de las áreas de la corteza y organizar la conducta humana.

Funcionalmente, se describen subcomponentes de las FE como la actualización, la planificación, la fluencia, la flexibilidad mental, la inhibición, el razonamiento, la memoria de trabajo, la toma de decisiones y la integración temporal (Fonseca, Rodríguez y Parra 2016) que permitirán una conducta eficaz y aceptada socialmente.

\section{Funciones ejecutivas y su base neuroanatómica}

Existe un consenso general para afirmar que las FE presentan como sustrato neuroanatómico principal la corteza prefrontal (Gutiérrez y Solis 2011; Lázaro y Solís 2008; Luria 1986; Stuss y Alexander 2000). En términos anatómicos, la corteza prefrontal (CPF) ocupa un lugar privilegiado para orquestar las FE.

La región frontal de la corteza cerebral se desarrolla en etapas filogenéticamente avanzadas. En el ser humano, la región frontal ocupa un tercio de toda la masa cortical, madura más tarde que el resto de las regiones corticales y presenta una amplia y variada conectividad neuronal (Betancur-Caro, Molina y Cañizales-Romaña 2016).

El área prefrontal es la máxima expresión de la inteligencia humana, ya que asume la responsabilidad de coordinar los procesos cognitivos, así como de programar la conducta para lograr la toma de decisiones. Se distinguen en él tres áreas funcionales: dorsolateral (CPDL), frontomedial (CFM) y orbitofrontal (COF) (Stuss 2001). Todas ellas tienen en común el control de las funciones ejecutivas, aunque cada una tiene competencias específicas. Mientras que las áreas dorsolaterales y cinguladas guardan más relación con los procesos cognitivos y la actividad mental superior, el área orbitaria tiene mayores implicaciones en el control y la regulación de los procesos emocionales.

\section{Funciones ejecutivas y su desarrollo evolutivo}

Las funciones ejecutivas comienzan su desarrollo en la infancia, cuando el niño o la niña adquieren la capacidad para controlar la conducta, usando información previa. Progresivamente el niño o la niña desarrollan mayor capacidad para resolver problemas complejos y para utilizar estrategias metacognoscitivas (Roselli 2003).

En un estudio de Korzeniowski (2011) sobre el desarrollo evolutivo del funcionamiento ejecutivo y su relación con el aprendizaje escolar, apunta a la existencia de tres periodos sensibles en el desarrollo de las áreas frontales: el primero se inicia entre los 4 y los 8 años de edad; el segundo, entre los 10 y 12 años y el tercero, entre los 15 y los 19 años.

\section{Funciones ejecutivas y rendimiento académico}

Las investigaciones centradas en la niñez se han interesado en el estudio de las FE debido a que estas se han relacionado con habilidades cognitivas, aprendizaje de la lectoescritura y competencias matemáticas (Aadland et al. 2018; Urrego Betancourt, Puerta Morales y Porto Torres 2016; Best, Miller y Naglieri 2011; Díez-Reviriego y Bausela-Herreras 2018; Fonseca, Rodríguez y Parra 2016; Golub, Rijavec y Olčar 2016; Mulder, Verhagen, Van der Ven, Slot y Leseman 2017; Zorza, Marino y Acosta Mesas 
2016). El rol de la función ejecutiva en el rendimiento académico en niños de 9 años demostró que las FE se incrementan ante su incursión en la etapa escolar.

Adquirir nuevas habilidades en el aula tiene mucho que ver con la forma en que los estudiantes se organizan, buscan y evalúan información (Risso et al. 2015), aspectos del pensamiento que dependen de las FE. Al inicio de la escolaridad se hace evidente el papel de la memoria en los procesos de aprendizaje que, al aumentar la exigencia académica, se apoyará en otros componentes de las FE para afrontar situaciones novedosas y tomar las decisiones adecuadas que demandarán las diferentes asignaturas escolares. Se incluyen para estos procesos la atención selectiva-sostenida, la memoria de trabajo, la memoria semántica, el lenguaje expresivo, la memoria, la planificación y la flexibilidad mental, que guardan estrecha relación con el aprendizaje escolar y el rendimiento en matemáticas, ciencias biológicas, idiomas y ciencias sociales.

El inicio o la vinculación a las actividades escolares se encuentra estrechamente relacionado con el concepto de rendimiento académico (RA), el cual se define como el grado de conocimiento obtenido por un alumno o alumna dentro del proceso de enseñanza-aprendizaje a través de una evaluación y cuyo resultado es expresado en una calificación que puede ser cualitativa o cuantitativa (Fonseca, Rodríguez y Parra 2016).

Considerando lo anteriormente mencionado, y con el respaldo de las diversas investigaciones descritas sobre la relación entre las FE y el RA, es importante que, desde los sistemas educativos y por medio de los profesionales de la educación, se cuente con elementos que permitan intervenir en las actividades académicas que fomenten el óptimo desarrollo de las FE (Blakey y Carroll 2015; Bodrova y Leong 2007; Raver et al. 2011).

Estas intervenciones estarían enfocadas en fortalecer los procesos relacionados con las FE que permitirán un mejor desempeño en lo académico en niños y niñas de educación primaria, partiendo de la posible relación encontrada entre las FE y el RA.

Los resultados obtenidos apoyan la importancia de analizar los factores ejecutivos relacionados con el ámbito académico, debido a que esta posible relación puede influir en el desarrollo y el perfeccionamiento de programas de refuerzo e intervención temprana que permiten identificar y fortalecer capacidades cognitivas, tanto en niños y niñas que presentan rendimiento normal como en quienes tienen rendimiento bajo (Fonseca, Rodríguez y Parra 2016).

El presente estudio tiene como objetivo realizar una revisión sistemática sobre el efecto de las FE en el RA a partir de las investigaciones publicadas al respecto durante las dos últimas décadas, con el fin de conocer el actual estado de la cuestión de dicho ámbito.

\section{Método}

Se ha llevado a cabo una revisión sistemática realizada de acuerdo a:

- La definición aportada por Higgins y Green (2008), en la que aquella se concibe como la revisión de una o varias cuestiones claramente formuladas, usando unos métodos sistemáticos y explícitos para identificar y seleccionar las investigaciones referentes a esta y analizar los datos de aquellos estudios incluidos en la revisión. 
- La declaración PRISMA (Preferred Reporting Items for Systematic Reviews and Meta-Analyses), cuya finalidad es evaluar la calidad de revisiones sistemáticas y metaanálisis (Moher et al. 2014).

\section{Estrategia de búsqueda}

Se ha realizado una búsqueda exhaustiva de la literatura en las bases de datos Dialnet, Pubmed y Proquest por proporcionar el acceso a un gran número de registros bibliográficos de artículos de revistas. También ofrecen referencias de publicaciones científicas de cualquier disciplina de conocimiento, ofreciendo una mirada transversal y multidisciplinar.

El periodo de recogida de artículos fue del 01.11.2017 al 01.11.2018. Se seleccionaron los años 2000 y 2018 por la tendencia creciente en estos años de las publicaciones dentro del ámbito educativo en relación con funciones ejecutivas y rendimiento académico.

Los descriptores utilizados para la búsqueda fueron funciones ejecutivas y rendimieto académico y Executive function y Academic performance para artículos en inglés.

En su búsqueda se utilizó el operador booleano $A N D$ para obtener documentos que contuvieran ambos términos de búsqueda.

La selección de los artículos fue realizada a través de las lecturas del título, resumen y palabras clave de cada trabajo.

\section{Criterios de selección}

Los criterios de inclusión que se incluyeron para la selección de los artículos fueron los siguientes:

- Trabajos empíricos y teóricos que aludían, bien en el título o en las palabras clave, a las funciones ejecutivas y el rendimiento académico (Executive function y Academic performance).

- Estudios publicados entre el 2000 y el 2018, ambos inclusive.

- Estudios publicados con un sistema de revisión por pares (peer review) para asegurar el estándar de calidad y fiabilidad.

\section{Procedimiento}

Con el fin de ilustrar el proceso, en la figura 1 se detalla el procedimiento seguido y las distintas fases llevadas a cabo en la revisión sistemática.

En el proceso de selección de los trabajos se tomó cada una de las bases de datos filtrando por año y descriptores y utilizando el operador boolear indicado. De esta manera, se identificaron 155 artículos.

Tras una revisión previa, se rechazaron 25 artículos por encontrarse duplicados. Posteriormente, se procedió a la revisión de los 130 estudios para valorar su posible inclusión, en base a la consulta de títulos, resúmenes y palabras clave. En esta fase se rechazaron 14 estudios por no cumplir los criterios de selección establecidos. 
Cabe señalar que, en los casos que habían ediciones dedicadas a las funciones ejecutivas, se revisaban los artículos completos, seleccionado solo aquellos que cumplían con los criterios de selección.

A continuación, se procedió a la lectura completa de los 116 artículos restantes, llevando a cabo una valoración de los estudios dicotómica y cualitativa, con calificaciones de aceptado o rechazado, de acuerdo con la presencia o ausencia de los criterios de selección definidos.

Durante este proceso, se rechazaron 48 estudios debido principalmente a que no se contextualizaban propiamente en el ámbito educativo, ni su foco principal se centraba en correlacionar las funciones ejecutivas con el rendimiento académico.

Finalmente se obtuvieron 68 estudios que sí cumplían con los criterios establecidos para la revisión sistemática.

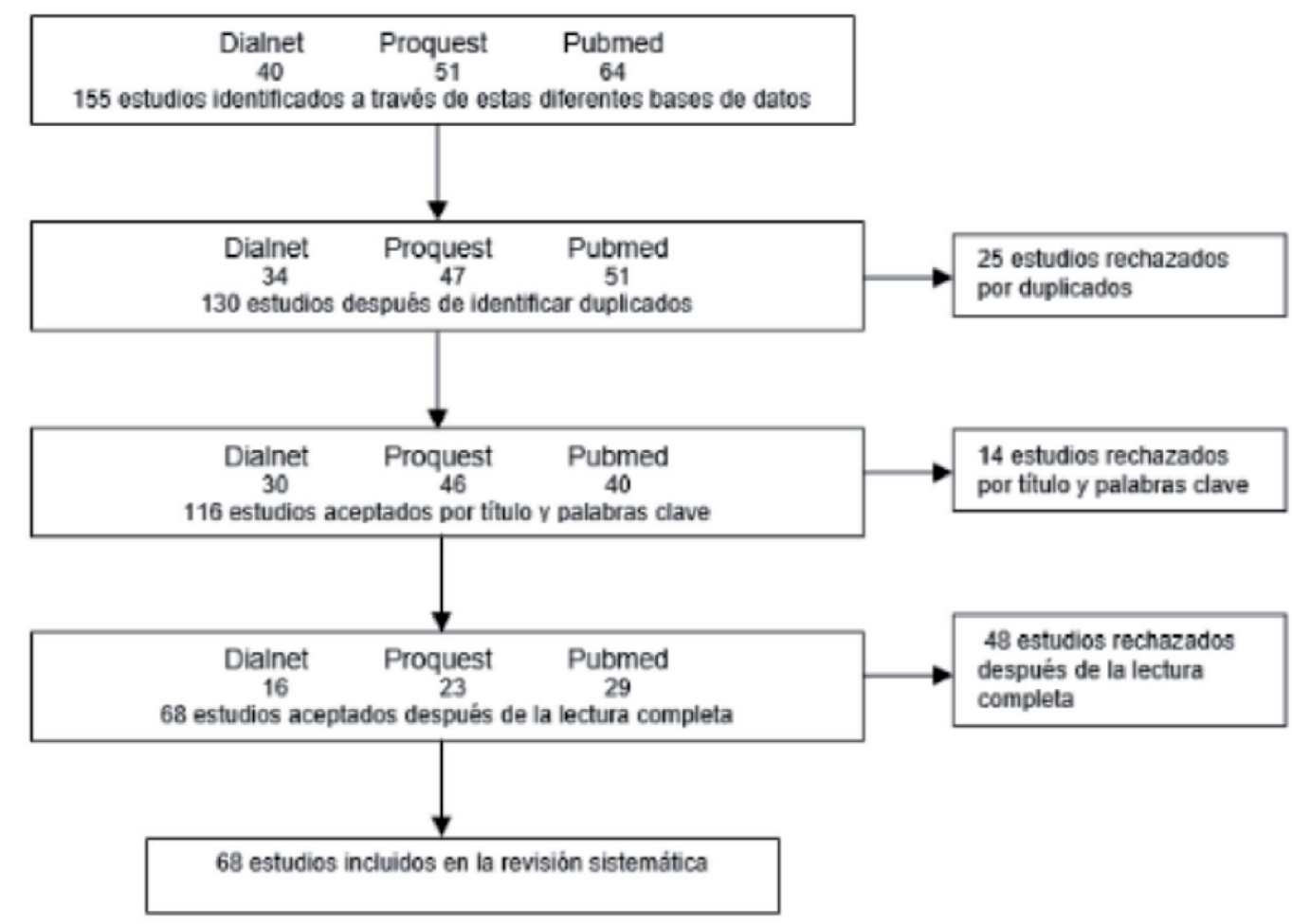

Figura 1. Diagrama de flujo de las fases de la revisión sistemática de acuerdo a la PRISMA.

Para el análisis y la extracción de datos, se tomaron las tres bases de datos y se extrajo la información de acuerdo a los siguientes ítems: a) título autor/es y año, $b$ ) palabras clave, $c$ ) centro investigativo o universidad en a que se realiza el estudio, $d$ ) tipo artículo, e) diseño metodológico, f) marco teórico y $g$ ) principales resultados y conclusiones.

Para su análisis, toda la información extraída de los artículos se organizó en cuadros, en base a los ítems anteriormente señalados. 


\section{Resultados}

\section{Producción de artículos en FE y RA}

Según se puede ver en la figura 2, la fluctuación en la producción de artículos publicados en los últimos diecinueve años ha aumentado considerablemente durante los últimos años y se observa un pico en los años 2016 y 2017.

Del total de trabajos seleccionados, el $43,28 \%$ han sido desarrollados en Europa, el $25,37 \%$ en America del Sur, el 19,50 \% en América del Norte, el 8,85\% en Asia y el $3 \%$ en Oceanía. Los países que más publicaciones tienen son España con 10 publicaciones e Inglaterra con 8.

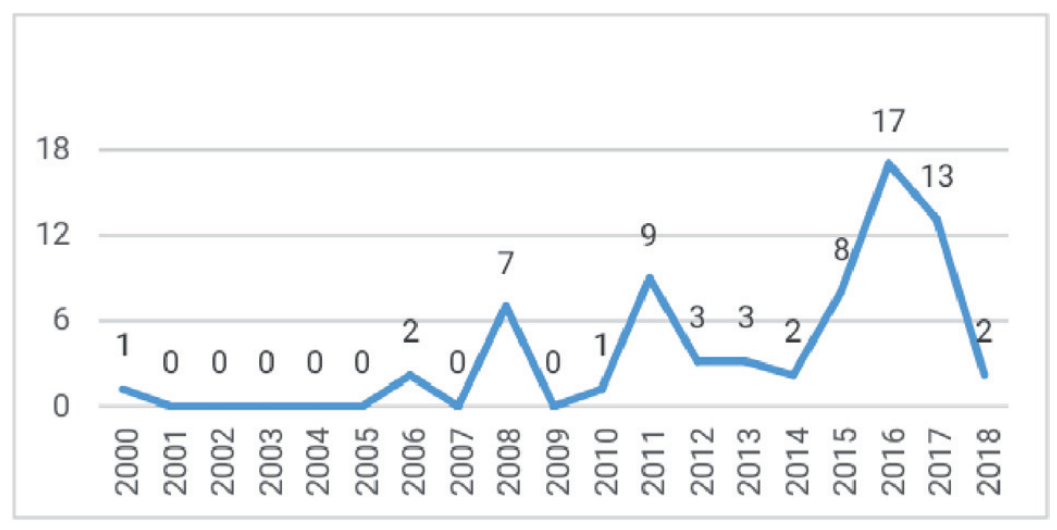

Figura 2. Producción de artículos en FE y RA.

¿Cómo y quién investiga en funciones ejecutivas y rendimiento académico?

¿Quién investiga en FE y RA? Se han identificado 194 autores entre los 68 artículos seleccionados. Estos autores provienen en su mayoría de universidades y centros de investigación adscritos a estas.

Por otra parte, la colaboración entre autores de departamentos de psicología es bastante frecuente y en algunos artículos es visible la participación de departamentos del área educativa. Se observó también participación de investigadores de universidades de países diferentes, sobre todo en estudios donde la muestra era intercultural. Centros de investigación en neuropsicología, neurociencia, cerebro y cognición son los más comunes.

Otro aspecto que se debe comentar en cuanto a los autores es que los estudios analizados han sido escritos en su mayoría por grupos de más de dos integrantes; solo se encontró un artículo publicado en solitario. Los demás artículos fueron trabajados en grupo. De los estudiós analizados, solo Feggy Ostrosky ha realizado un total de 3 publicacions en el periodo estudiado.

Respecto al cómo se investiga sobre FE y RA, se presentan dos dimensiones: el tipo de estudio y el diseño del mismo. En cuanto al tipo de estudio, se observa que el 65,67\% son trabajos empíricos y el $34,33 \%$ son teóricos. Respecto al diseño en los estudios empíricos, el $64,10 \%$ de los estudios han utilizado el correlacional, el $20,54 \%$ son descriptivos y el $15,36 \%$ son logitudinales. Los estudios teóricos constiuyen en su mayoría revisiones de la literatura. 
Tabla 1

Principales hallazgos en el estudio de FE y RA

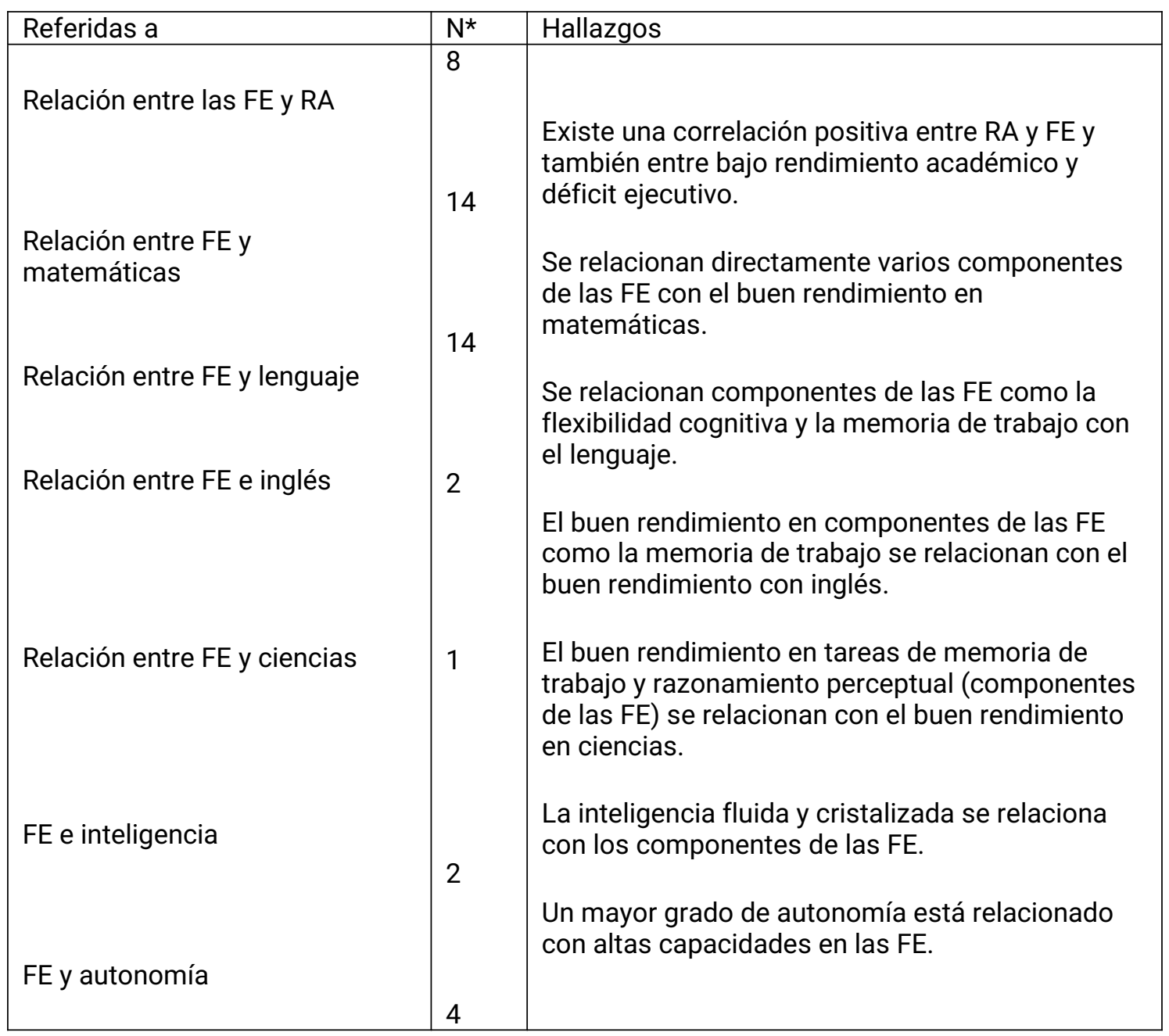

*Número de artículos que tienen el hallazgo citado

En la tabla 1 se recogen los principales hallazgos reflejados en los artículos analizados, así como el número de artículos que tratan cada tema. Así, se han usado seis criterios para clasificarlos, referidos a: 1) relación entre $F E$ y $R A, 2$ ) relación entre FE y matemáticas, 3) relación entre FE y lenguaje, 4) relación entre FE e inglés, 5) relación entre FE y ciencias, 6) FE e inteligencia y 7) FE y auntonomía .

\section{Discusión y conclusiones}

El presente estudio de revisión aporta una perspectiva del desarrollo de la investigación sobre FE y RA, centrada en las bases de datos de Proquest, Dialnet y Pubmed, recogiendo los principales hallazgos en este campo.

Al respecto, cabe destacar la denominada «Década del cerebro» (Jones y Mendell 1999), en donde los avances tecnológicos en imágenes in vivo del cerebro llevaron a que la neurociencia educativa fuese viable y, con ella, la apertura de nuevas líneas de investigación, como el estudio de las FE y RA. Debido a esto, los años de mayor 
producción en este tema se encuentran en la última década, principalmente entre los años 2016 y 2017.

Sin embargo, y aunque ha existido una evolución favorable en cuanto al volumen de investigaciones que abordan esta temática en los últimos años, es complejo resaltar a algún investigador o grupo de investigación con una producción reseñable, lo que ratifica el estado investigativo inicial en este campo.

A la luz de estos resultados, hemos de prestar atención a la escasez de estudios teóricos que pueden incidir en la base conceptual de esta área de estudio y que puede conllevar errores en la práctica educativa. Este aspecto actualmente ha dejado lugar a controversias y errores prácticos que deben solucionarse con la realización de un número mayor de estudios teóricos que permitan consolidar un cuerpo de trabajo que forge las bases teóricas necesarias para llevar al campo educativo estrategias viables y sólidas.

La amplia producción de tipo empírico ha utilizado en su mayoría un diseño correlacional, mientras que los estudios teóricos han empleado revisiones de literatura. Por tanto, una vía para promover la extracción de conclusiones más rigurosas y generalizables sería incrementar el número de estudios experimentales con diseños transeccionales, decriptivos y longitudinales (Hernández, Fernández y Baptista 2006) que permitan seguir aportando diferentes perspectivas al conocimiento de este fenómeno.

Los principales hallazgos referentes a la investigación en FE y RA son alentadores, pues la gran mayoría de estudios han demostrado que existe una estrecha relación entre estos dos constructos. Sin embargo, este campo de la neurociencia educativa tiene ante sí algunos retos importantes, como dar respuesta a cuestiones relativas a cómo se desarrollan las FE a través de la vida y, en concreto, si todos los componentes de las FE aparecen en los primeros años de vida o si cada uno se presenta en un estadío específico del desarrollo. La respuesta a estas preguntas transformaría la investigación en FE y RA, pues establecería unos límites claros de qué y cuándo se debe estudiar en el contexto educativo.

Otro aspecto encontrado que requiere un mayor estudio es conocer si todos los componentes de las FE están relacionados con todas las asignaturas o si existen ciertos procesos de las FE relacionados con asignaturas específicas.

Asímismo, es importante aclarar cuáles son los límites y las diferencias entre el concepto de inteligencia y el de FE, para cerrar el debate que se ha dejado ver desde el surgimiento de este constructo.

Finalmente, un campo que destaca por su relevancia constatada en múltiples investigaciones es el de la autonomía y su efecto positivo en el desarrollo de las FE. Todos los aspectos antes mencionados se encuentran en un momento de investigación prematuro y requieren aunar un mayor número de trabajos que permitan llegar a conclusiones más concretas y fortalezcan este promisorio campo de investigación.

De las conclusiones obtenidas se puede decir que las FE podrían ser un buen componente a tener en cuenta en el curriculo escolar y que se deben fortalecer transversalmente en todas las asignaturas, dada la relación demostrada con el RA. Queda mucho camino por recorrer, pero estudios de revisión como el presentado en este trabajo pueden ayudar a delimitar el camino a seguir. 
Una de las limitaciones que presenta este estudio es que se centra en determinadas bases de datos, por lo que sería necesario ampliar el número de dichas bases que permita un análisis más exhaustivo de los estudios llevados a cabo en este campo.

\section{Referencias bibliográficas}

Aadland, Katrine Nyvoll, Eivind Aadland, John Roger Andersen, Arne Lervåg, Vegard Fusche Moe, Geir Kåre Resaland y Yngvar Ommundsen. 2018. «Executive function, behavioral self-regulation, and school related well-being did not mediate the effect of school-based physical activity on academic performance in numeracy in 10-year-old children. The Active Smarter Kids (ASK) study». Frontiers in psychology, 9: 245.

Best, John R., Patricia H. Miller y Jack A. Naglieri. 2011. «Relations between executive function and academic achievement from ages 5 to 17 in a large, representative national sample». Learning and individual differences, 21(4): 327-336.

Betancur-Caro, María Luisa, David A. Molina y Lilian Yohana Cañizales-Romaña. 2016. «Entrenamiento Cognitivo de las Funciones Ejecutivas en la Edad Escolar». Revista Latinoamericana de Ciencias Sociales, Niñez y Juventud, 14(1): 359-368.

Blakey, Emma y Daniel J. Carroll. 2015. «A short executive function training program improves preschoolers' working memory». Frontiers in psychology, 6: 1827.

Bodrova, Elena y Deborah J. Leong. 2007. Tools of the Mind: The Vygotskian Approach to Early childhood education. Columbus, $\mathrm{OH}$ : Merrill/Prentice Hall.

Díez-Reviriego, Ester y Esperanza Bausela-Herreras. 2018. «Funciones ejecutivas y la competencia para resolver problemas matemáticos en Educación Primaria». Cuadernos de Neuropsicología/Panamerican Journal of Neuropsychology, 12(1): 42-57.

Fonseca, Gina Paola, Lucía Carlota Rodríguez y Javier Humberto Parra. 2016. «Relación entre funciones ejecutivas y rendimiento académico por assignatures en escolares de 6 a 12 años». Hacia la promoción de la salud, 21(2): 41-58.

Golub, Tajana Ljubin, Majda Rijavec y Diana Olčar. 2016. "The relationship between executive functions and flow experiences in learning». Studia psychologica, 58(1): 47-58.

Gutiérrez, Asucena Lozano y Feggy Ostrosky Solís. 2011. «Desarrollo de las Funciones Ejecutivas y de la Corteza Prefrontal». Revista Neuropsicología, Neuropsiquiatría y Neurociencias, 11(1): 159-172.

Hernández, Roberto, Carlos Fernández y Pilar Baptista. 2006. Metodología de la investigación. México: McGraw-Hill.

Higgins, Julian P. T. y Sally Green. 2008. Cochrane handbook for systematic reviews of interventions. West Sussex, England: Cochrane Collaboration \& Wiley.

Jones, Edward G. y Lorne M. Mendell. 1999. "Assessing the decade of the brain». Science, 284: 739.

Korzeniowski, Celina Graciela. 2011. «Desarrollo evolutivo del funcionamiento ejecutivo y su Relación con el aprendizaje escolar». Revista de Psicología Universidad Católica de Argentina, 7(13): 7-26.

Lázaro, Julio César F. y Feggy Ostrosky Solís. 2008. «Neuropsicología de lóbulos frontales, funciones ejecutivas y conducta humana». Revista neuropsicología, neuropsiquiatría y neurociencias, 8(1): 47-58.

Lezak, Muriel Deutsch. 1983. Neuropsychological assessment. New York: Oxford.

Luria, Alexander R. 1986. Las funciones corticales superiores del hombre. México: Fontamara. 
Moher, David, Alessandro Liberati, Jennifer Tetzlaff, Douglas G. Altman y PRISMA Group. 2014. «Ítems de referencia para publicar Revisiones sistemáticas y Metaanálisis: La Declaración PRISMA». Revista Española de Nutrición Humana y Dietética, 18(3): 172-181.

Mulder, Hanna, Josje Verhagen, Sanne H G. Van der Ven, Pauline L. Slot y Paul P. Leseman. 2017. "Early executive function at age two predicts emergent mathematics and literacy at age five». Frontiers in psychology, 8: 1706.

Raver, Cybele, Stephanie M. Jones, Christine Li-Grining, Fuhua Zhai, Kristen Bubb y Emily Pressler. 2011. "CSRP's impact on low-income preschoolers' preacademic skills: self-regulation as a mediating mechanism». Child Development, 82: 362-378.

Risso, Alicia, Manuel García, Montserrat Durán, Juan Carlos Brenlla, Manuel Peralbo y Alfonso Barca. 2015. «Un análisis de las relaciones entre funciones ejecutivas, lenguaje y habilidades matemáticas». Revista de Estudios e Investigación en Psicología y Educación, 9: 73-78.

Roselli, Mónica. 2003. "Maduracion cerebral y desarrollo cognoscitivo». Revista Latinoamericana de Ciencias Sociales, Niñez y Juventud, 1(1): 125-144.

Stuss, Donald T. 1992. «Biological and physiological development of executive function». Brain and Cognition, 20: 8-23.

-. 2001. «Stroop performance in focal lesions patients: dissociation of processess amd frontal lobe lesion location». Neuropsycologia, 39: 771-786. Doi: 10.1016/S0028-3932(01)00013-6.

Stuss, Donald T. y Michael P. Alexander. 2000. «Executive Functions and the frontal lobes: a conceptual view». Psychology Research, 63: 289-298.

Urrego Betancourt, Yaneth, Laura Puerta Morales y María Fernanda Porto Torres. 2016. «Funciones ejecutivas de estudiantes de básica primaria en dos regiones de Colombia». Cuadernos de Neuropsicología, 10(3): 77-90.

Zorza, Juan Pablo, Julián Marino y Alberto Acosta Mesas. 2016. «Executive functions as predictors of school performance and social relationships: primary and secondary school students». The Spanish journal of psychology, 19: 1-10. 